\title{
HAMILTONIAN FLOWS ON STATIONARY MANIFOLDS
}

\author{
Allan P. Fordy and Simon D. Harris \\ Dedicated to Martin Kruskal to celebrate his 70th birthday.
}

\begin{abstract}
We consider the important question of the relationship between an "integrable" nonlinear evolution equation, a partial differential equation (PDE), and its stationary reduction, an ordinary differential equation (ODE). We are mainly concerned with "transporting" algebraic machinery from the PDE to the ODE. In particular, given a Hamiltonian representation of the original PDE, we would like to derive the Poisson brackets, Hamiltonians, etc. for the stationary flow.

In this paper, we give a systematic derivation of these Hamiltonian properties, using an $x-t$ reversed form of the spectral problem for the PDE. We obtain, in this way, the Hamiltonian structures, etc. of the PDE, but written in terms of the stationary manifold coordinates. These structures naturally reduce to their finite dimensional counterparts for the stationary flow.

We illustrate our results with several examples, including the Korteweg-de Vries, dispersive water wave, and Ito hierarchies.
\end{abstract}

\section{Introduction}

When Martin Kruskal and his collaborators discovered the soliton and introduced the Inverse Scattering Method for solving the Korteweg-de Vries (KdV) equation, they opened up a whole new field which exploded into numerous subfields having repercussions far beyond the subject of nonlinear wave equations. One of the early discoveries was that the $\mathrm{KdV}$ equation can be regarded as a completely integrable, infinite dimensional Hamiltonian system [14,23]. This gave a whole new impetus to the general theory of completely integrable Hamiltonian systems, (both infinite and finite dimensional). During the 19th Century, there had been great interest in such systems, but the subject fell from grace when Bruns and Poincaré showed the nonintegrability of the 3-body problem. However, following the discovery of the KdV's Hamiltonian properties, many new examples were found, such as the Toda lattice, Calogero-Moser system, and numerous partial differential equations (PDEs). At the end of the 1970's, two very important discoveries were made.

1. Bogoyavlenskii and Novikov [7] showed that each of the stationary reductions of the $\mathrm{KdV}$ hierarchy constitutes a completely integrable (finite dimensional) Hamiltonian system.

2. Magri [18] and Gelfand and Dorfman [15] independently developed the theory of "bi-Hamiltonian" systems, giving the KdV equation as a prime example.

Received December 27, 1995, revised November 25, 1996.

1991 Mathematics Subject Classification: 58F05, 58F07.

Key words and phrases: complete integrability, stationary flow. 
The first of these is concerned with stationary flows, meaning that we consider solutions $u(x, t)$ (for example) of a PDE (generally a system) which are independent of $t$. Such solutions satisfy an ordinary differential equation (ODE) (generally a system). In simple cases, such as the $\mathrm{KdV}$ equation, this is obviously a Hamiltonian system (even Newtonian). Generally, this is highly non-trivial and follows (in our context) from the Hamiltonian property of the PDE. As noted in (1) above, this Hamiltonian system of ODEs can actually be completely integrable.

The "second" (local) Hamiltonian structure of the KdV equation (noted in (2) above) is perhaps best understood in the context of Miura's "Remarkable explicit nonlinear transformation" [19], which is, in fact, a Poisson map, under which the second Poisson bracket of the KdV equation is just the image of the single (local) Poisson bracket of the modified KdV (MKdV) equation. These ideas were generalised in the 1980s, being developed for general Lax and many other classes of equations $[11,12,17,3]$.

In [5], it was shown that the stationary flows of the KdV and MKdV hierarchies are also bi-Hamiltonian. Thus, we have examples of bi-Hamiltonian PDEs whose stationary flows are also bi-Hamiltonian. However, as has often been pointed out (see, for example, [8]), the situation is unsatisfactory, in that the relationship between structures (such as Poisson brackets) associated with a PDE and those associated with its stationary reduction is rather obscure. Ideally, we would like to "transport" known machinery from an infinite dimensional system, directly to its finite dimensional reductions. However, the original approach of Bogoyavlenskii and Novikov required going out of the Lax pair/zero curvature framework and using a Lagrangian representation to start afresh. Whilst the Lagrangian itself is derived from a PDE Hamiltonian density, the resulting Poisson bracket bears no resemblance to that of the PDE. The situation was improved by Antonowicz and Blaszak [1], who used $x-t$ reversal (see the discussion leading to equation (24) below) together with the Miura map (following the approach of [5]).

In this paper, we give a systematic treatment of this problem, by starting with a zero curvature formulation of the given PDE in $x-t$ reversed coordinates. Using techniques employed in [3], we systematically derive (from a knowledge of the zerocurvature representation) a Hamiltonian formulation of the PDE in $x-t$ reversed coordinates (our examples are, in fact, bi- or tri-Hamiltonian). The resulting infinite dimensional Poisson brackets naturally reduce to their finite dimensional counterparts for the stationary flows. The basic approach is to rearrange the Lax or zero curvature equation in an appropriate way. In the case of a "generic" spectral problem, this is straightforward, but for our stationary flows, which are deep reductions of the generic case, the calculation is highly non-trivial.

In Section 2, we briefly describe how to build Hamiltonian operators from a zero curvature representation, using the approach of $[3,4]$. We first describe the generic case, illustrating these ideas with the KdV hierarchy. For the benefit of the nonspecialist, we use the latter as a vehicle for introducing a number of "well-known" ideas and facts, such as Miura maps and the construction of conserved densities. This section also contains some important background information on stationary flows and the idea of $x-t$ reversal. We refer the reader to [4] for a more formal and complete exposition of all these ideas. 
The main results of this paper are in Section 3, where we show how to construct the Hamiltonian structure of a PDE in terms of any coordinate system on the stationary manifold. The main difficulty here stems from the fact that the zero curvature representation is a deep reduction of the generic case. After some general discussion, we consider the case of the stationary $\mathrm{KdV}$ equation. Whilst this example is suitable for introducing a number of ideas, it is too special. The stationary $5^{\text {th }}$-order KdV equation is much more representative, so it is studied next in this section.

In Section 4, we consider some systems of PDEs associated with "energy dependent" Schrödinger operators. In particular, we discuss the dispersive water wave (DWW) and Ito hierarchies. In these cases, it is possible to use the recursion operator of the PDE to derive a third Hamiltonian structure. Whilst this construction would be invalid in the stationary reduction, the resulting Poisson brackets do reduce correctly.

\section{The zero curvature representation in Hamiltonian form}

Since the early discoveries of Gardner, Greene, Kruskal, and Miura, there has been interest in equations with a so-called Lax or zero curvature representation. We consider nonlinear evolution equations which can be represented as the integrability conditions of a pair of linear equations:

$$
\begin{aligned}
& \Psi_{x}=U \Psi, \\
& \Psi_{t}=V \Psi
\end{aligned}
$$

where $U$ and $V$ are $N \times N$ matrices, dependent both upon the functions entering the nonlinear equations and upon a spectral parameter $\lambda$. The integrability conditions are

$$
U_{t}-V_{x}+[U, V]=0 .
$$

This can be interpreted as the zero curvature condition for the Lie algebra-valued connection defined by (1) and (2). With $\lambda_{t}=0$, this is said to define an isospectral flow of the spectral problem (1). For a given $U$, the matrix $V$ can be determined algorithmically for simple classes of $\lambda$-dependence. We write (3) in the form

$$
U_{t}=(\partial-\operatorname{ad} U) V \quad \text { with } \partial \equiv \partial_{x}
$$

where $(\operatorname{ad} U) V=[U, V]$. These can be represented with respect to some basis, with $U=u_{i} e_{i}$ :

$$
\sum_{i} u_{i t} e_{i}=-\left(\sum_{i, j} B_{i j} e_{i} \otimes e_{j}\right)\left(\sum_{k} v_{k}^{*} e_{k}^{*}\right)=-\sum_{i, j}\left(B_{i j} v_{j}^{*}\right) e_{i}
$$

with $e_{i}^{*}$ the dual basis of $e_{i}$, so that $B_{i j} e_{i} \otimes e_{j}$ is a $(2,0)$ tensor whose components are (at most) first-order differential operators. In components,

$$
u_{i t}=-B_{i j} v_{j}^{*}, \quad v_{i}^{*}=g_{i j} v_{j}, \quad g_{i j}=\operatorname{tr}\left(e_{i} e_{j}\right) .
$$

Here the matrix $\mathbf{B}$ (components $B_{i j}$ ) is the Hamiltonian operator and the vector $\left(v_{1}^{*}, \ldots, v_{N}^{*}\right)^{T}$ is the gradient of some Hamiltonian functional. The simplest way of calculating this is to check the trace form $\operatorname{tr}\left(U_{t} V\right)$ since

$$
\operatorname{tr}\left(U_{t} V\right)=\mathbf{u}_{t} \cdot \mathbf{v}^{*} \text {. }
$$

We are mainly interested in the hierarchy of isospectral flows of a spectral problem with $U$ dependent upon $\lambda$ and the "potential functions" in some specified way. We 
often have polynomial dependence upon $\lambda$, generating a sequence of polynomial flows, labeled by "times" $t_{m}$ :

$$
U_{t_{m}}-V_{(m) x}+\left[U, V_{(m)}\right]=0 .
$$

All the examples of this paper involve second-order spectral problems, so we just present the case $N=2$ here (see [16] for some higher order examples).

Example 2.1 ( $2 \times 2$ matrices). We have

$$
U=\left(\begin{array}{cc}
a & b \\
c & -a
\end{array}\right), \quad V=\left(\begin{array}{cc}
\gamma & \alpha \\
\beta & -\gamma
\end{array}\right)
$$

and define

$$
\mathbf{u}=(b, c, a)^{T}, \mathbf{v}=(\alpha, \beta, \gamma)^{T}, \quad \text { giving } \quad \mathbf{v}^{*}=(\beta, \alpha, 2 \gamma)^{T}=-\delta_{u} h
$$

The above formula gives

$$
\mathbf{B}=\left(\begin{array}{ccc}
0 & -\partial+2 a & -b \\
-\partial-2 a & 0 & c \\
b & -c & -\frac{1}{2} \partial
\end{array}\right)
$$

With some $\lambda$-dependence of $a, b$, and $c$, these can form blocks within larger Hamiltonian structures. In [9], this is used to generate multi-Hamiltonian structures. When $U$ is linear in $\lambda$, we get a bi-Hamiltonian formulation.

Example 2.2 (The linear pencil: $w=\lambda+w_{0}, q=q_{0}, r=r_{0}$ ). This is the case of the generalised Zakharov-Shabat (ZS) spectral problem, which immediately gives the biHamiltonian formulation

$$
u_{t}=\left(\mathbf{B}_{1}-\lambda \mathbf{B}_{0}\right) \delta h, \quad \delta h \equiv\left(\delta_{q_{0}} h, \delta_{r_{0}} h, \delta_{w_{0}} h\right)^{T},
$$

written explicitly as

$$
\left(\begin{array}{c}
q_{0} \\
r_{0} \\
w_{0}
\end{array}\right)_{t}=\left[\left(\begin{array}{ccc}
0 & -\partial+2 w_{0} & -q_{0} \\
-\partial-2 w_{0} & 0 & r_{0} \\
q_{0} & -r_{0} & -\frac{1}{2} \partial
\end{array}\right)-\lambda\left(\begin{array}{ccc}
0 & -2 & 0 \\
2 & 0 & 0 \\
0 & 0 & 0
\end{array}\right)\right]\left(\begin{array}{c}
\beta \\
\alpha \\
2 \gamma
\end{array}\right)
$$

This example is deceptively simple as a consequence of the simple dependence upon $\lambda$. The spectral problems associated with stationary flows are deep reductions of the generic case and have complicated $\lambda$-dependence.

2.1. The KdV hierarchy. If, in example (2.1), we put $a=0, b=1, c=\frac{1}{4} \lambda-u$, corresponding to the Schrödinger equation $\psi_{1 x x}+u \psi_{1}=\frac{1}{4} \lambda \psi_{1}$, then we find

$$
\gamma=-\frac{1}{2} \alpha_{x}, \quad \beta=-\frac{1}{2} \alpha_{x x}-\left(u-\frac{1}{4} \lambda\right) \alpha,
$$

and

$$
u_{t}=\left(\mathbf{B}_{1}-\lambda \mathbf{B}_{0}\right) \alpha
$$

where

$$
\mathbf{B}_{0}=\frac{1}{2} \partial, \quad \mathbf{B}_{1}=\frac{1}{2} \partial^{3}+2 u \partial+u_{x}
$$


In a simple way, this has generated the bi-Hamiltonian formulation of the KdV hierarchy, with $\alpha=\delta_{u} \mathcal{H}$. Taking $\alpha$ to be a polynomial expansion in $\lambda$ gives an element of the usual KdV hierarchy. The first three polynomial flows are $u_{t_{0}}=u_{x}$ and

$$
\begin{aligned}
& u_{t_{1}}=u_{x x x}+6 u u_{x}, \\
& u_{t_{2}}=u_{x x x x x}+10 u u_{x x x}+20 u_{x} u_{x x}+30 u^{2} u_{x},
\end{aligned}
$$

which correspond, respectively, to $\alpha_{(0)}=1, \alpha_{(1)}=\lambda+2 u$, and $\alpha_{(2)}=\lambda^{2}+2 \lambda u+$ $2 u_{x x}+6 u^{2}$.

The Miura map. The second Hamiltonian structure $\mathbf{B}_{1}$ is the image of a much simpler structure through the Miura map. By adding a parameter, we get Gardner's generalisation, which enables us to obtain both Hamiltonian structures and an infinite number of conservation laws. Explicitly, this takes the form

$$
u=M[v] \equiv-v_{x}-v^{2}+\frac{1}{4} \lambda
$$

The Jacobian (Fréchet derivative) of this map is defined by $M^{\prime}[v] w=\left.\frac{d}{d \varepsilon} M[v+\varepsilon w]\right|_{\varepsilon=0}$, giving $M^{\prime}=-\partial-2 v$. As in the finite dimensional case, the Jacobian is used to "transport" Poisson brackets (now infinite dimensional). Generally, with

$$
v_{t}=\tilde{\mathbf{B}} \delta_{v} \tilde{\mathcal{H}}
$$

where $\tilde{\mathbf{B}}$ is some Hamiltonian operator, and $u=M[v]$, we have

$$
u_{t}=M^{\prime} v_{t} \quad \text { and } \quad \delta_{v} \tilde{\mathcal{H}}=\left(M^{\prime}\right)^{\dagger} \delta_{u} \mathcal{H}
$$

where $\tilde{\mathcal{H}}[v]$ and $\mathcal{H}[u]$ are related by $\tilde{\mathcal{H}}=\mathcal{H} \circ M(\bmod \operatorname{Im} \partial)$ and $\left(M^{\prime}\right)^{\dagger}$ is the formal adjoint of $M^{\prime}$. Piecing these formulae together, we get

$$
u_{t}=M^{\prime} \tilde{\mathbf{B}}\left(M^{\prime}\right)^{\dagger} \delta_{u} \mathcal{H} .
$$

In this way, the single (local) Hamiltonian structure of the MKdV hierarchy, $-\frac{1}{2} \partial$, is transported to give

$$
M^{\prime}\left(-\frac{1}{2} \partial\right)\left(M^{\prime}\right)^{\dagger}=\frac{1}{2} \partial^{3}+2 u \partial+u_{x}-\frac{1}{2} \lambda \partial \equiv \mathbf{B}_{1}-\lambda \mathbf{B}_{0} .
$$

Each Hamiltonian functional, $\mathcal{H}[u]$, is pulled back to a functional (an equivalence class of functionals) of $v$ :

$$
\tilde{\mathcal{H}}[v]=\mathcal{H}[M[v]]
$$

and we have the mapping of Hamiltonian vector fields

$$
v_{t}=-\frac{1}{2} \partial \delta_{v} \tilde{\mathcal{H}} \quad \mapsto \quad u_{t}=\left(\mathbf{B}_{1}-\lambda \mathbf{B}_{0}\right) \delta_{u} \mathcal{H}
$$

The Miura map can be used to build an infinite family of commuting Hamiltonians. We introduce the asymptotic expansion

$$
v=-\frac{1}{2} k+u k^{-1}+\sum_{i=2}^{\infty} v_{i} k^{-i}, \quad \lambda=k^{2},
$$


which starts as

$$
\begin{array}{ll}
v_{2}=u_{x}, & v_{3}=u^{2}+u_{x x} \\
v_{4}=\left(2 u^{2}+u_{x x}\right)_{x}, & v_{5}=2 u^{3}-u_{x}^{2}+\left(u_{x x x}+6 u u_{x}\right)_{x},
\end{array}
$$

and satisfies (for $i \geq 1$ )

$$
v_{i+1}=v_{i x}+\sum_{j=0}^{i} v_{j} v_{i-j}
$$

so, at each level, $v_{i+1}$ can be solved in terms of a local functional of $v_{1}, \ldots, v_{i}$. It is easy to prove that the even coefficients $v_{2 i}$ are exact derivatives, so that

$$
\delta_{u} v=k^{-1}\left(1+2 u \lambda^{-1}+\left(2 u_{x x}+6 u^{2}\right) \lambda^{-2}+\cdots\right) .
$$

If we define $\mathcal{H}_{n}=v_{2 n+1}, n=0,1, \ldots$, we get $\delta_{u} v=k^{-1} \sum_{n=0}^{\infty} \lambda^{-n} \delta_{u} \mathcal{H}_{n}$. Since

$$
\left(\mathbf{B}_{1}-\lambda \mathbf{B}_{0}\right) \delta_{u} v=-\frac{1}{2} M^{\prime} \partial \delta_{v} v=0
$$

we may define $\alpha=k \delta_{u} v=\sum_{n=0}^{\infty} \lambda^{-n} \delta_{u} \mathcal{H}_{n}$, and the $m^{\text {th }}$ flow by

$$
u_{t_{m}}=\left(\mathbf{B}_{1}-\lambda \mathbf{B}_{0}\right) \alpha_{(m)}, \quad \alpha_{(m)}=\left(\lambda^{m} \alpha\right)_{+}
$$

where the subscript "+" means taking only the non-negative powers of $\lambda$. This gives the bi-Hamiltonian representation

$$
u_{t_{m}}=\mathbf{B}_{1} \delta_{u} \mathcal{H}_{m}=\mathbf{B}_{0} \delta_{u} \mathcal{H}_{m+1} .
$$

This bi-Hamiltonian ladder property guarantees that the Hamiltonians $\mathcal{H}_{n}$ are in involution with respect to both Poisson brackets. The flow (14) is the image of the vector field

$$
v_{t_{m}}=-\frac{1}{2} \partial \beta_{(m)}, \quad \beta_{(m)}=\left.\left(M^{\prime}\right)^{\dagger} \alpha_{(m)}\right|_{u=M[v]} .
$$

The first two flows and their "modifications" are

$$
\begin{aligned}
& \alpha_{(0)}=1, \quad \beta_{(0)}=-2 v \quad \Rightarrow \quad u_{t_{0}}=u_{x}, \quad v_{t_{0}}=v_{x} \\
& \left.\begin{array}{l}
\alpha_{(1)}=\lambda+2 u, \\
\beta_{(1)}=-3 \lambda v+4 v^{3}-2 v_{x x}
\end{array}\right\} \Rightarrow\left\{\begin{array}{l}
u_{t_{1}}=u_{x x x}+6 u u_{x}, \\
v_{t_{1}}=v_{x x x}-6 v^{2} v_{x}+\frac{3}{2} \lambda v_{x} .
\end{array}\right.
\end{aligned}
$$

For each flow (15), $-\frac{1}{2} \beta_{(m)}$ is an expression in $v$ and its $x$-derivatives. By substituting (13) into (15) and equating coefficients of $k$, we obtain an infinite sequence of conservation laws, obeyed by each member of the hierarchy

$$
\mathcal{H}_{n t_{m}}=\mathcal{F}_{n m x} \text {. }
$$

The fluxes $\mathcal{F}_{n m}$ play the role of conserved densities when we consider the $x-t_{m}$ reversal in later sections. The above discussion of the Miura map and its uses is very important for what follows. These ideas and methods have a wide range of applicability in the theory of integrable systems (see [4] for a review). 
Stationary reductions. Bogoyavlenskii and Novikov [7] used a generalised Legendre transformation to write the stationary flows of the KdV hierarchy in Hamiltonian form and then proved complete integrability. In [5], the Miura map was used to construct a second Hamiltonian structure, and in [1], the equations were viewed with $x$ and $t$ reversing their roles.

The $m^{\text {th }}$-stationary flow in the KdV hierarchy is defined by

$$
u_{t_{m}}=\frac{1}{2} \partial \delta_{u} \mathcal{H}_{m+1}=0, \quad m \geq 1,
$$

which is in the form of a generalised Lagrangian equation (see the Appendix)

$$
\delta_{u} \mathcal{L}_{m+1}=0, \quad \mathcal{L}_{m+1}=\mathcal{H}_{m+1}-c_{m} u .
$$

Remark 2.1. Not all operators have such a simple kernel. For the KdV hierarchy, Ker $\mathbf{B}_{0}=\delta_{u}(c u)$, but Ker $\mathbf{B}_{1}$ is not so simple. For $\mathbf{B}_{1}$, however, it is possible to extend the space of variables in order to achieve this [6]. Generally, constants such as $c$ above arise from the kernel of the relevant Hamiltonian operator $\mathbf{B}$ (see the examples of Section 4).

It is possible to write these Lagrangian equations as an $m$-degrees of freedom canonical Hamiltonian system. If we take $c_{m}$ as a dynamical variable, then the Poisson bracket is degenerate, with $c_{m}$ as Casimir:

$$
\{f, g\}=\frac{\partial f}{\partial x_{i}} J_{i j} \frac{\partial g}{\partial x_{j}}=(\nabla f)^{T} J(\nabla g), \quad J=\left(\begin{array}{ccc}
0 & I_{m} & 0 \\
-I_{m} & 0 & 0 \\
0 & 0 & 0
\end{array}\right)
$$

where $I_{m}$ is the $m \times m$ unit matrix. Bogoyavlenskii and Novikov [7] showed that this Hamiltonian system is completely integrable with first integrals given by the fluxes of the Hamiltonians $\mathcal{H}_{1}, \ldots, \mathcal{H}_{m}$ :

$$
\begin{aligned}
& 0=\mathcal{H}_{0 t_{m}}=\mathcal{F}_{0 m x}, \\
& 0=\mathcal{H}_{k t_{m}}=\mathcal{F}_{k m x} \quad k=1, \ldots, m,
\end{aligned}
$$

where the first of these is just the equation, which is an ODE of order $2 m$. This is used to eliminate higher derivatives from the fluxes $\mathcal{F}_{k m}$, which then can be written as functions of the variables $\left(q_{i}, p_{i}, c_{m}\right): \mathcal{F}_{k m}=h_{k}\left(q_{i}, p_{i}, c_{m}\right), k=1, \ldots, m$. The later fluxes become functionally dependent upon $h_{1}, \ldots, h_{m}$. Since $\left\{H_{i}, H_{m}\right\}=0$ for all elements of the KdV hierarchy, the stationary manifold is invariant under the action of the flows. The first components of each of the commuting Hamiltonian flows of the stationary flow are precisely

$$
u_{t_{k}}=\frac{1}{2} \partial \delta \mathcal{H}_{k+1}, \quad k=1, \ldots, m-1,
$$

when written in terms of the stationary manifold coordinates, since $q_{1}=u$.

The Miura map between the KdV and MKdV equations induces a diffeomorphism between corresponding stationary manifolds. The fluxes/Hamiltonians for the stationary flows are calculated directly from

$$
v_{t_{m}}=-\frac{1}{2} \tilde{c}_{m x}
$$

with $\tilde{c}_{m}$ given as a differential polynomial in $v$. Thus, substituting the expansion for $v$ into $\tilde{c}_{m}$, we directly construct the fluxes as coefficients in the k-expansion of $\tilde{c}_{m}$. 
Furthermore, it is possible to use the Miura map to construct the second Hamiltonian structure for the stationary flows.

Example 2.3 (The stationary KdV equation). The stationary KdV equation (which defines $c_{1}$ ) is

$$
2 u_{x x}+6 u^{2}=c_{1}
$$

with Lagrangian $\mathcal{L}$ given by $\mathcal{L}=2 u^{3}-u_{x}^{2}-c_{1} u$ and canonical coordinates

$$
q=u, \quad p=-2 u_{x}, \quad c_{1},
$$

$c_{1}$ being the Casimir of the degenerate canonical bracket. The Legendre transformation gives

$$
h_{\mathcal{L}}=c_{1} q-2 q^{3}-\frac{1}{4} p^{2}
$$

which is the flux of $\mathcal{H}_{1}=u^{2}$.

The Miura map induces the diffeomorphism $M$ given by

$$
\begin{aligned}
q & =\frac{1}{4} \lambda-\frac{1}{2} \tilde{p}-\tilde{q}^{2}, \\
p & =-\tilde{c}_{1}+4 \tilde{q}^{3}+2 \tilde{q} \tilde{p}-3 \lambda \tilde{q}, \\
c_{1} & =\frac{1}{2} \tilde{p}^{2}-2 \tilde{q}^{4}+2 \tilde{q} \tilde{c}_{1}+3 \lambda \tilde{q}^{2}+\frac{3}{8} \lambda^{2},
\end{aligned}
$$

relating (17) to the stationary MKdV equation

$$
-2 v_{x x}+4 v^{3}-3 \lambda v=\tilde{c}_{1},
$$

which has Lagrangian $\mathcal{L}=v_{x}^{2}+v^{4}-\tilde{c}_{1} v-\frac{3}{2} \lambda v^{2}$ and canonical coordinates

$$
\tilde{q}=v, \quad \tilde{p}=2 v_{x}, \quad \tilde{c}_{1} .
$$

The canonical Poisson brackets between the modified variables

$$
\{\tilde{q}, \tilde{p}\}=1, \quad\left\{\tilde{q}, \tilde{c}_{1}\right\}=\left\{\tilde{p}, \tilde{c}_{1}\right\}=0
$$

induce

$$
\{q, p\}=-2 q-\lambda, \quad\left\{q, c_{1}\right\}=-p, \quad\left\{p, c_{1}\right\}=-2 c_{1}+12 q^{2} .
$$

This is a non-canonical Poisson bracket for the stationary KdV equation. In fact, it is a one-parameter family of Poisson brackets; the coefficient of $\lambda$ is just the canonical bracket, while the remaining part is the second bracket [5]. This proves not only that each coefficient is independently a Poisson bracket, but also that they are compatible.

The variable $c_{1}$ is a Casimir for the degenerate canonical bracket with its level surfaces being symplectic leaves. The symplectic leaves of the second Poisson bracket are different, being the level surfaces of $h_{\mathcal{L}}$, while $c_{1}$ now generates the stationary KdV equation. 
Reversing the role of $x$ and $t$. The KdV equation (10) defines a flow on the infinite dimensional space with coordinates $\left(u, u_{x}, u_{x x}, \ldots\right)$ and evolution parameter $t$. Since we are interested in the stationary flow, we consider the KdV equation (a PDE) as a special flow on the infinite dimensional space with coordinates $\left(q, p, c_{1}, q_{t}, p_{t}, c_{1 t}, \ldots\right)$ where $q, p$, and $c_{1}$ are defined by (17)-(18), and with evolution parameter $x$. We now must give the first three components of this flow:

$$
\left(\begin{array}{c}
q \\
p \\
c_{1}
\end{array}\right)_{x}=\left(\begin{array}{c}
-\frac{1}{2} p \\
6 q^{2}-c_{1} \\
2 q_{t}
\end{array}\right)
$$

In [1], this flow was shown to be Hamiltonian, with a $3 \times 3$ matrix Poisson bracket, which reduces to the stationary case when the potential functions are independent of $t$ :

$$
\left(\begin{array}{c}
q \\
p \\
c_{1}
\end{array}\right)_{x}=\left(\begin{array}{ccc}
0 & 1 & 0 \\
-1 & 0 & 0 \\
0 & 0 & 2 \partial_{t}
\end{array}\right)\left(\begin{array}{c}
c_{1}-6 q^{2} \\
-\frac{1}{2} p \\
q
\end{array}\right)
$$

where this last vector is just $\delta_{s}\left(c_{1} q-2 q^{3}-\frac{1}{4} p^{2}\right)$ where $\delta_{s} h=\left(\delta_{q} h, \delta_{p} h, \delta_{c_{1}} h\right)^{T}$ denotes the gradient of $h$ with respect to the stationary coordinates. This could have been done in any coordinate system, generally producing a non-canonical Poisson bracket. However, with the same canonical variables as before, the equation took "canonical" form.

Similarly, the MKdV equation (see (16)) can be written

$$
\left(\begin{array}{c}
\tilde{q} \\
\tilde{p} \\
\tilde{c}_{1}
\end{array}\right)_{x}=\left(\begin{array}{ccc}
0 & 1 & 0 \\
-1 & 0 & 0 \\
0 & 0 & -2 \partial_{t}
\end{array}\right)\left(\begin{array}{c}
\tilde{c}_{1}-4 \tilde{q}^{3}+3 \lambda \tilde{q} \\
\frac{1}{2} \tilde{p} \\
\tilde{q}
\end{array}\right)=\hat{\mathbf{B}} \delta_{\tilde{s}} \tilde{h}
$$

where $\tilde{h}=\tilde{q} \tilde{c}_{1}-\tilde{q}^{4}+\frac{1}{4} \tilde{p}^{2}+\frac{3}{2} \lambda \tilde{q}^{2}$, and $\delta_{\tilde{s}}$ denotes the gradient with respect to the modified stationary coordinates.

The Miura map (19) is only changed by $-2 \tilde{q}_{t}$ in the definition of $c_{1}$. As shown in [1], the above bracket for the MKdV equation then is transformed to

$$
\left(\begin{array}{ccc}
0 & -2 q & \partial_{t}-p \\
2 q & -2 \partial_{t} & 12 q^{2}-2 c_{1} \\
\partial_{t}+p & 2 c_{1}-12 q^{2} & 4\left(q \partial_{t}+\partial_{t} q\right)
\end{array}\right)-\lambda\left(\begin{array}{ccc}
0 & 1 & 0 \\
-1 & 0 & 0 \\
0 & 0 & 2 \partial_{t}
\end{array}\right)
$$

These define Poisson brackets for the full $\mathrm{KdV}$ equation written in terms of the stationary manifold coordinates and clearly reduce to those (see (21)) of the stationary equations when the potential functions are independent of $t$.

Such $x-t$ reversed flows and Poisson brackets also have been considered in $[13,20]$.

\section{The $x-t$ reversed spectral problem}

The above derivation of (24) was ad hoc, suited only to simple examples. We now fit our stationary flows into the scheme described in Section 2 and thus treat them as isospectral flows. In so doing, we give a systematic construction of Hamiltonian structures, Hamiltonians, and time evolutions of the "wave functions" (of the associated spectral problem). For the latter, we give a formula in terms of gradients of the corresponding Hamiltonians. In this section, we mainly present this in the context of 
the KdV hierarchy, but start with some general statements. Other examples are dealt with in the next section and in [16].

Consider an equation in some hierarchy with zero curvature representation (6). For simplicity of notation, we restrict attention to scalar equations, but multi-component examples present no difficulty (see Section 4). In order to consider the $t_{m}$ stationary flow, we just reverse the roles of $U$ (which equals $V_{(0)}$ ) and $V_{(m)}$ (which now is playing the role of the spectral problem). We write the zero curvature equations as

$$
V_{(m) x}=U_{\xi}-\left[V_{(m)}, U\right]
$$

where $\xi=t_{m}$. It should be noted (see the examples) that $V_{(m)}$ depends upon $u$ and some (but not too many) of its $x$-derivatives. Following the argument which led to (22), we write this in terms of some set of coordinates on the stationary manifold. For comparison of results, we choose the canonical coordinates.

We write $V_{(m)}$, when written in terms of the stationary manifold coordinates, as $\mathcal{U}$ and consider a general evolution (labeled by $\tau$ ) with zero curvature representation

$$
\mathcal{U}_{\tau}=\mathcal{V}_{\xi}-[\mathcal{U}, \mathcal{V}]
$$

The particular choice $\mathcal{V}=U=V_{(0)}$ corresponds to our original equation in $x-t$ reversed form (in terms of the stationary manifold coordinates). All the examples of this paper are associated with $2 \times 2$ matrix problems, so we now restrict to this case (see [16] for other cases). We have

$$
\mathcal{U}=\left(\begin{array}{cc}
a & b \\
c & -a
\end{array}\right), \quad \mathcal{V}=\left(\begin{array}{cc}
\gamma & \alpha \\
\beta & -\gamma
\end{array}\right)
$$

where $a, b$, and $c$ are functions of $q_{i}, p_{i}, c_{i}$, and $\lambda$. Except for very simple cases (such as the $\mathrm{KdV}$ equation), there are more than 3 coordinates, so (25) does not give the full evolution $\mathbf{q}_{\tau}$ where $\mathbf{q}=\left(q_{1}, \ldots, q_{n}, p_{1}, \ldots, p_{n}, c_{1}, \ldots, c_{k}\right)^{T}$. Indeed, if we represent $\mathcal{U}$ by the vector $\mathbf{b}=(b, c, a)^{T}$, we obtain

$$
\mathbf{b}_{\tau}=\tilde{\mathbf{B}} \delta_{b} \tilde{h}
$$

where $\tilde{\mathbf{B}}$ is given by (7) (with $\partial \equiv \frac{\partial}{\partial \xi}$ ) and $\delta_{b} \tilde{h}$ is shorthand for

$$
\left(\frac{\delta \tilde{h}}{\delta b}, \frac{\delta \tilde{h}}{\delta c}, \frac{\delta \tilde{h}}{\delta a}\right)^{T}=-(\beta, \alpha, 2 \gamma)^{T}
$$

If $\mathcal{J}_{\varphi}$ denotes the Fréchet derivative of the map $\varphi: \mathbf{q} \mapsto \mathbf{b}$, then

$$
\mathbf{b}_{\tau}=\mathcal{J}_{\varphi} \mathbf{q}_{\tau} \quad \text { and } \quad \delta_{q} h=\mathcal{J}_{\varphi}^{\dagger} \cdot \delta_{b} \tilde{h}
$$

where $h=\tilde{h} \circ \varphi$. Our task is to resolve (27) and (28) into the form

$$
\mathbf{q}_{\tau}=\mathbf{B} \delta_{q} h
$$

even though $\mathcal{J}_{\varphi}$ is a $3 \times(2 n+k)$ matrix, with right kernel of dimension $2 n+k-3$.

This construction deals with each stationary manifold separately. 
3.1. The $\mathrm{KdV}$ equation. In this case, we have example 2.1 with (8):

$$
\mathcal{U} \equiv V_{(1)}=\left(\begin{array}{cc}
\frac{1}{2} p & \lambda+2 q \\
\frac{1}{4} \lambda^{2}-\frac{1}{2} \lambda q+q^{2}-\frac{1}{2} c_{1} & -\frac{1}{2} p
\end{array}\right)
$$

where coordinates $\left(q, p, c_{1}\right)$ are defined by (17)-(18). The map $\varphi$ then has Jacobian

$$
\mathcal{J}_{\varphi}=\left(\begin{array}{ccc}
2 & 0 & 0 \\
2 q-\frac{1}{2} \lambda & 0 & -\frac{1}{2} \\
0 & \frac{1}{2} & 0
\end{array}\right)
$$

In this case, it is possible to invert $\mathcal{J}_{\varphi}$ to obtain

$$
\mathbf{q}_{\tau}=\mathcal{J}_{\varphi}^{-1} \tilde{\mathbf{B}}\left(\mathcal{J}_{\varphi}^{-1}\right)^{\dagger} \delta_{q} h=\mathbf{B} \delta_{q} h
$$

where

$$
\mathbf{B}=\left(\begin{array}{ccc}
0 & -2 q-\lambda & -p+\partial \\
2 q+\lambda & -2 \partial & 12 q^{2}-2 c_{1} \\
p+\partial & -12 q^{2}+2 c_{1} & (4 q-\lambda) \partial+\partial(4 q-\lambda)
\end{array}\right)
$$

As previously mentioned, this is related to the simple Hamiltonian structure of (23) through the Miura map. This is an alternative way of proving that $\mathbf{B}$ is indeed Hamiltonian.

To calculate the hierarchy of Hamiltonians, we repeat the argument used with the $\mathrm{KdV}$ hierarchy in the usual coordinates. Since $\tilde{c}_{1}$ is a flux in the usual MKdV language, it is now a conserved density under $\tau$-evolutions. Furthermore, it is a Casimir function of the Poisson bracket given in (23), so it generates the bi-Hamiltonian ladder (24)

$$
\hat{\mathbf{B}} \delta_{\tilde{q}} \tilde{c}_{1}=0 \Rightarrow \mathbf{B} \delta_{q} \tilde{c}_{1}=M^{\prime} \hat{\mathbf{B}}\left(M^{\prime}\right)^{\dagger} \delta_{q} \tilde{c}_{1}=M^{\prime} \hat{\mathbf{B}} \delta_{\tilde{q}} \tilde{c}_{1}=0
$$

where $\mathbf{B}$ is given by (30). The asymptotic series for $\tilde{c}_{1}$ can be derived from the known series (13) by substitution into the formula (20), replacing $x$-derivatives of $u$ by $q, p$, $c_{1}$, and their $\xi$-derivatives. Explicitly, we have

$$
\begin{aligned}
\tilde{q} & =-\frac{1}{2} k+q k^{-1}-\frac{1}{2} p k^{-2}+\left(\frac{1}{2} c_{1}-2 q^{2}\right) k^{-3}+\left(q_{\xi}+q p\right) k^{-4}+\cdots \\
\tilde{c}_{1} & =-2 v_{x x}+4 v^{3}-3 \lambda v \\
& =k^{3}-c_{1} k^{-1}-2 q_{\xi} k^{-2}+\left(\frac{1}{2} p^{2}+4 q^{3}-2 c_{1} q+p_{\xi}\right) k^{-3}+\cdots
\end{aligned}
$$

The Hamiltonians

$$
h_{-1}=\frac{1}{2} c_{1}, \quad h_{0}=-\frac{1}{4} p^{2}+q c_{1}-2 q^{3}, \quad h_{1}=\frac{1}{4} c_{1}^{2}+q_{\xi} p
$$


and second Hamiltonian structure, generate

$$
\begin{gathered}
\left(\begin{array}{l}
q \\
p \\
c_{1}
\end{array}\right)_{\tau_{-1}}=\left(\begin{array}{c}
-\frac{1}{2} p \\
6 q^{2}-c_{1} \\
2 q_{\xi}
\end{array}\right),\left(\begin{array}{c}
q \\
p \\
c_{1}
\end{array}\right)_{\tau_{0}}=\left(\begin{array}{l}
q \\
p \\
c_{1}
\end{array}\right)_{\xi}, \\
\left(\begin{array}{c}
q \\
p \\
c_{1}
\end{array}\right)_{\tau_{1}}=\left(\begin{array}{c}
\frac{1}{2} c_{1 \xi}-2 q q_{\xi}-\frac{1}{2} p c_{1} \\
-2 q_{\xi \xi}-2 q p_{\xi}+6 q^{2} c_{1}-c_{1}^{2} \\
\left(4 c_{1} q-4 q^{3}-\frac{1}{2} p^{2}-p_{\xi}\right)_{\xi}
\end{array}\right) .
\end{gathered}
$$

In general, we have

$$
\mathbf{q}_{\tau_{r}}=\left(\mathbf{B}_{1}-\lambda \mathbf{B}_{0}\right) \delta_{q} h_{(r)}=\mathbf{B}_{1} \delta_{q} h_{r}, \quad h_{(r)}=\lambda^{r+1} h_{-1}+\cdots+h_{r}
$$

The time evolution $\Psi_{\tau_{r}}$ is given in terms of $\delta_{q} h_{(r)}$ :

$$
\gamma=-\frac{\delta h_{(r)}}{\delta p}, \quad \alpha=2 \frac{\delta h_{(r)}}{\delta c_{1}}, \quad \beta=-\frac{1}{2} \frac{\delta h_{(r)}}{\delta q}+\left(\frac{1}{2} \lambda-2 q\right) \frac{\delta h_{(r)}}{\delta c_{1}} .
$$

Thus $h_{-1}$ generates the KdV equation and $h_{0}$ the "translational flow", telling us that $\tau_{0}=\xi$. We can use the $\tau_{-1}$ flow to write $p, c_{1}$ and $q_{\xi}$ in terms of $q$ and its $\tau_{-1}$ derivatives after which

$$
q_{\tau_{1}}=q_{x x x x x}+10 q q_{x x x}+20 q_{x} q_{x x}+30 q^{2} q_{x}
$$

where we have written $\tau_{-1}=x$.

Thus, the hierarchy is just the KdV hierarchy in disguise. However, without the $\tau_{-1}$ flow, this hierarchy shows no hint of being reducible from the $\left(q, p, c_{1}\right)$ space to the $q$ space. In these coordinates, the reduction to the stationary flow $q_{\xi}=0$ is very natural, with (30) reducing to (21) and the bi-Hamiltonian ladder becoming finite.

\subsection{The fifth-order $\mathrm{KdV}$ equation. Here}

$$
u_{t_{2}}=\frac{1}{2} \partial \delta \mathcal{H}_{3}=0, \quad \mathcal{H}_{3}=u_{x x}^{2}-10 u u_{x}^{2}+5 u^{4} .
$$

The Lagrangian $\mathcal{L}_{3}=u_{x x}^{2}-10 u u_{x}^{2}+5 u^{4}-c_{2} u$ gives the canonical variables

$$
\begin{aligned}
& q_{1}=u, \quad q_{2}=u_{x}, \quad p_{2}=2 u_{x x}, \quad p_{1}=-2 u_{x x x}-20 u u_{x} \\
& c_{2}=2 u_{x x x x}+20 u u_{x x}+10 u_{x}^{2}+20 u^{3}
\end{aligned}
$$

with $c_{2}$ being a Casimir function of the degenerate canonical bracket. The spectral problem is given by $V_{(2)}$ of the $\mathrm{KdV}$ hierarchy (example 2.1 with (8) and $\alpha=\alpha_{(2)}$ ), with (32):

$$
\Psi_{\xi}=\mathcal{U} \Psi, \quad \text { where } \quad \mathcal{U}=\left(\begin{array}{cc}
a & b \\
c & -a
\end{array}\right)
$$


with $a, b, c$ given by

$$
\begin{aligned}
& a=-\lambda q_{2}+\frac{1}{2} p_{1}+4 q_{1} q_{2}, \\
& b=\lambda^{2}+2 \lambda q_{1}+p_{2}+6 q_{1}^{2}, \\
& c=\frac{1}{4} \lambda^{3}-\frac{1}{2} \lambda^{2} q_{1}-\frac{1}{4} \lambda\left(p_{2}+2 q_{1}^{2}\right)-\frac{1}{2} c_{2}+q_{1} p_{2}+4 q_{1}^{3}-q_{2}^{2} .
\end{aligned}
$$

We denote this mapping by $\varphi: \mathbf{q} \mapsto \mathbf{b}$. As before, we consider an evolution $\mathcal{V}$ (see (26)) where $\alpha, \beta$, and $\gamma$ are functions of $\left(q_{i}, p_{i}, c_{2}\right)$, their $\xi$-derivatives, and $\lambda$. The integrability conditions are again (25).

The Jacobian $\mathcal{J}_{\varphi}$ is the $3 \times 5$ matrix

$$
\mathcal{J}_{\varphi}=\left(\begin{array}{ccccc}
12 q_{1}+2 \lambda & 0 & 0 & 1 & 0 \\
12 q_{1}^{2}+p_{2}-\lambda q_{1}-\frac{1}{2} \lambda^{2} & -2 q_{2} & 0 & q_{1}-\frac{1}{4} \lambda & -\frac{1}{2} \\
4 q_{2} & 4 q_{1}-\lambda & \frac{1}{2} & 0 & 0
\end{array}\right),
$$

which does not, therefore, have a unique inverse. Thus the equation

$$
\mathbf{b}_{\tau}=\mathcal{J}_{\varphi} \mathbf{q}_{\tau}
$$

is underdetermined for the components of $\mathbf{q}_{\tau}$, whilst the equation

$$
\delta_{q} h=\mathcal{J}_{\varphi}^{\dagger} \delta_{b} \tilde{h}
$$

gives an overdetermined system of equations for the components of $\delta_{b} \tilde{h}$, which we would like to solve in terms of $\delta_{q} h$. Thus $\delta_{q} h$ must be orthogonal to $\mathbf{w} \in \operatorname{Ker} \mathcal{J}_{\varphi}$

$$
\mathbf{w}^{\dagger} \delta_{q} h=\left(\mathcal{J}_{\varphi} \mathbf{w}\right)^{\dagger} \delta_{b} \tilde{h} \Rightarrow \mathbf{w}^{\dagger} \delta_{q} h=0,
$$

apparently giving rise to some constraints on $\delta_{q} h$. In fact, these "constraints" can be identified with part of the recursion relation of the bi-Hamiltonian ladder, so do not constitute a restriction. The (right) kernel of $\mathcal{J}_{\varphi}$ is important in what follows, and is spanned by

$$
\begin{aligned}
& \mathbf{v}_{1}=\left(1,0,-8 q_{2},-12 q_{1}-2 \lambda, 2 p_{2}\right)^{T} \\
& \mathbf{v}_{2}=\left(0,1,-8 q_{1}+2 \lambda, 0,-4 q_{2}\right)^{T} .
\end{aligned}
$$

The left kernel of $\mathcal{J}_{\varphi}$ is $\mathbf{0}$. We then can solve (uniquely) for $\delta_{b} \tilde{h}$ :

$$
\delta_{a} \tilde{h}=2 \delta_{p_{1}} h, \quad \delta_{b} \tilde{h}=\delta_{p_{2}} h+\left(2 q_{1}-\frac{1}{2} \lambda\right) \delta_{c_{2}} h, \quad \delta_{c} \tilde{h}=-2 \delta_{c_{2}} h,
$$

with "constraints"

$$
\begin{aligned}
& \lambda \delta_{p_{1}} h=-\frac{1}{2} \delta_{q_{2}} h+4 q_{1} \delta_{p_{1}} h+2 q_{2} \delta_{c_{2}} h \\
& \lambda \delta_{p_{2}} h=\frac{1}{2} \delta_{q_{1}} h-4 q_{2} \delta_{p_{1}} h-6 q_{1} \delta_{p_{2}} h+p_{2} \delta_{c_{2}} h .
\end{aligned}
$$

Since we do not have a left inverse for $\mathcal{J}_{\varphi}$, we cannot use (29). We write (25) as

$$
\mathcal{J}_{\varphi} \mathbf{q}_{\tau}=\tilde{\mathbf{B}} \delta_{b} \tilde{h}
$$


where $\tilde{\mathbf{B}}$ is the $3 \times 3$ Hamiltonian structure given in (27). We write (36) as $\delta_{b} \tilde{h}=D \delta_{q} h$ where

$$
D=\left(\begin{array}{ccccc}
0 & 0 & 0 & 1 & 2 q_{1}-\frac{1}{2} \lambda \\
0 & 0 & 0 & 0 & -2 \\
0 & 0 & 2 & 0 & 0
\end{array}\right)
$$

but are free to add the transpose of an element of the right kernel of $\mathcal{J}_{\varphi}$

$$
\delta_{b} \tilde{h}=\left(D+\sum_{i=1}^{2} \mathbf{f}_{i} \mathbf{v}_{i}^{\dagger}\right) \delta_{q} h \equiv \hat{D} \delta_{q} h .
$$

Indeed,

$$
\mathcal{J}_{\varphi}^{\dagger} \delta_{b} \tilde{h}=\delta_{q} h \quad \Rightarrow \quad \hat{D} \mathcal{J}_{\varphi}^{\dagger} \delta_{b} \tilde{h}=\hat{D} \delta_{q} h=\delta_{b} \tilde{h} \quad \Rightarrow \quad \hat{D} \mathcal{J}_{\varphi}^{\dagger}=I_{3}
$$

where $I_{3}$ is the $3 \times 3$ identity matrix. Thus, for any choice of $\mathbf{f}_{i}, \hat{D}$ is a left inverse of $\mathcal{J}_{\varphi}^{\dagger}$.

Whilst the $\mathbf{f}_{i}$ make no difference to the formula (36) for $\delta_{b} \tilde{h}$, they can change the right-hand side of

$$
\mathcal{J}_{\varphi} \mathbf{q}_{\tau_{r}}=\tilde{\mathbf{B}}\left(D+\sum_{i=1}^{2} \mathbf{f}_{i} \mathbf{v}_{i}^{\dagger}\right) \delta_{q} h_{(r)}
$$

which involves the truncated Hamiltonian $h_{(r)}$, which no longer satisfies the infinite recursion relation represented by the "constraint" (37).

We now use our knowledge of the standard KdV hierarchy. The Hamiltonian $h_{(r)}=$ $\left(\lambda^{r+2} h\right)_{+}$is given in terms of the fluxes previously calculated:

$$
h_{-2}=\frac{1}{2} c_{2}, \quad h_{-1}=-5 q_{1}^{4}+10 q_{1} q_{2}^{2}+q_{1} c_{2}+q_{2} p_{1}+\frac{1}{4} p_{2}^{2} .
$$

We have the following correspondence between the usual $\mathrm{KdV} t_{k}$ and $\tau_{m}$ :

$$
\begin{aligned}
& t_{0}=x, t_{1}, \quad t_{2}=\xi, \quad t_{3}, \ldots \\
& \tau_{-2}=x, \tau_{-1}, \quad \tau_{0}=\xi, \quad \tau_{1}, \ldots
\end{aligned}
$$

The $\tau_{-2}$ flow is just the $x-t_{2}$ reversed fifth-order $\mathrm{KdV}$ equation

$$
\left(\begin{array}{c}
q_{1} \\
q_{2} \\
p_{1} \\
p_{2} \\
c_{2}
\end{array}\right)_{\tau_{-2}}=\left(\begin{array}{c}
q_{2} \\
\frac{1}{2} p_{2} \\
20 q_{1}^{3}-10 q_{2}^{2}-c_{2} \\
-20 q_{1} q_{2}-p_{1} \\
2 q_{1 \xi}
\end{array}\right),
$$


whilst the $\tau_{-1}$ flow is the $\mathrm{KdV}$ equation for $q_{1}$ and its prolongation to the other variables (but written in these coordinates)

$$
\left(\begin{array}{c}
q_{1} \\
q_{2} \\
p_{1} \\
p_{2} \\
c_{2}
\end{array}\right)_{\tau_{-1}}=\left(\begin{array}{c}
-4 q_{1} q_{2}-\frac{1}{2} p_{1} \\
-10 q_{1}^{3}-2 q_{1} p_{2}+q_{2}^{2}+\frac{1}{2} c_{2} \\
120 q_{1}^{4}+30 q_{1}^{2} p_{2}+20 q_{1} q_{2}^{2}-6 q_{1} c_{2}+4 q_{2} p_{1}+p_{2}^{2}-2 q_{2 \xi} \\
20 q_{1}^{2} q_{2}+4 q_{1} p_{1}-2 q_{2} p_{2}+2 q_{1 \xi} \\
12 q_{1} q_{1 \xi}+p_{2 \xi}
\end{array}\right)
$$

Substituting these equations into (40) determines $f_{i}$ to be zero. Thus, for this example, the correct choice of $\hat{D}$ is $D$.

Remark 3.1. Whilst for the KdV hierarchy and several other examples, we find $\mathbf{f}_{i}=0$, this is not always true (in this paper, see Ito's equation).

We wish to find a $5 \times 5$ matrix $\mathbf{B}$ such that

$$
\tilde{\mathbf{B}}=\mathcal{J}_{\varphi} \mathbf{B} \mathcal{J}_{\varphi}^{\dagger}
$$

Using $\hat{D}(=D)$, we can find a particular solution $\overline{\mathbf{B}}$ of (41) satisfying

$$
\overline{\mathbf{B}}=D^{\dagger} \tilde{\mathbf{B}} D \quad \text { so that } \quad \mathcal{J}_{\varphi} \overline{\mathbf{B}} \mathcal{J}_{\varphi}^{\dagger}=\left(\mathcal{J}_{\varphi} D^{\dagger}\right) \tilde{\mathbf{B}}\left(D \mathcal{J}_{\varphi}^{\dagger}\right)=\tilde{\mathbf{B}}
$$

This $\overline{\mathbf{B}}$ explicitly takes the form

$$
\overline{\mathbf{B}}=\left(\begin{array}{ccccc}
0 & 0 & 0 & 0 & 0 \\
0 & 0 & 0 & 0 & 0 \\
0 & 0 & -2 \partial & \Gamma_{3} & \Gamma_{1} \\
0 & 0 & -\Gamma_{3} & 0 & 2 \partial+\Gamma_{2} \\
0 & 0 & -\Gamma_{1} & 2 \partial-\Gamma_{2} & 4\left(\partial q_{1}+q_{1} \partial\right)-2 \lambda \partial
\end{array}\right)
$$

where

$$
\begin{aligned}
& \Gamma_{1}=40 q_{1}^{3}+8 q_{1} p_{2}-4 q_{2}^{2}-2 c_{2}-2 \lambda p_{2} \\
& \Gamma_{2}=-16 q_{1} q_{2}-2 p_{1}+4 \lambda q_{2} \\
& \Gamma_{3}=12 q_{1}^{2}+2 p_{2}+4 \lambda q_{1}+2 \lambda^{2}
\end{aligned}
$$

To this we can add any skew-symmetric matrix which is in the kernel of $M \mapsto \mathcal{J}_{\varphi} M \mathcal{J}_{\varphi}^{\dagger}$ :

$$
\mathbf{B}=\overline{\mathbf{B}}+\sum_{i, j=1}^{2}\left(\mathbf{a}_{i} \mathbf{v}_{i}^{\dagger}-\mathbf{v}_{i} \mathbf{a}_{i}^{\dagger}\right)
$$

We wish to write the evolution equations for $\mathbf{q}$ as 


$$
\mathbf{q}_{\tau_{r}}=\mathbf{B} \delta_{q} h_{(r)}=\left(\overline{\mathbf{B}}+\sum_{i, j=1}^{2}\left(\mathbf{a}_{i} \mathbf{v}_{i}^{\dagger}-\mathbf{v}_{i} \mathbf{a}_{i}^{\dagger}\right)\right) \delta_{q} h_{(r)}
$$

but also have (38) after replacing $\tilde{\mathbf{B}}$ :

$$
\mathcal{J}_{\varphi}\left(\mathbf{q}_{\tau_{r}}-\overline{\mathbf{B}} \delta_{q} h_{(r)}\right)=0
$$

To be consistent, we require

$$
\mathcal{J}_{\varphi} \sum_{i, j=1}^{2}\left(\mathbf{a}_{i} \mathbf{v}_{i}^{\dagger}-\mathbf{v}_{i} \mathbf{a}_{i}^{\dagger}\right) \delta_{q} h_{(r)}=0
$$

Since we do not have $\mathbf{v}_{i}^{\dagger} \delta_{q} h_{(r)}=0$, we need $\mathcal{J}_{\varphi} \mathbf{a}_{i}=0$, so that $\mathbf{a}_{i}=\sum_{j=1}^{2} \mathbf{v}_{j} d_{j i}$, and

$$
\mathbf{B}=\overline{\mathbf{B}}+\sum_{i, j=1}^{2} \mathbf{v}_{i} b_{i j} \mathbf{v}_{j}^{\dagger}
$$

where $b_{i j}=(\mathbf{b})_{i j}$ with $\mathbf{b}$ a skew-adjoint matrix differential operator (in the present case, a simple skew-symmetric matrix). Equation (43) implies $\mathbf{q}_{\tau_{r}}-\overline{\mathbf{B}} \delta_{q} h_{(r)} \in \operatorname{Ker} \mathcal{J}_{\varphi}$. From the forms of $\overline{\mathbf{B}}$ and $\mathbf{v}_{i}$, we have explicitly

$$
\mathbf{q}_{\tau_{r}}=\overline{\mathbf{B}} \delta_{q} h_{(r)}+\mathbf{v}_{1} q_{1 \tau_{r}}+\mathbf{v}_{2} q_{2 \tau_{r}}
$$

Matching (42) and (44), we have

$$
q_{i \tau_{r}}=\sum_{j=1}^{2} b_{i j} \mathbf{v}_{j}^{\dagger} \delta_{q} h_{(r)}, \quad i=1,2
$$

The coordinates (32) define the fifth-order $\mathrm{KdV}$ equation as an $x-t$ reversed flow, with $\tau_{-2}=x$, from which we find

$$
q_{1 \tau_{-2}}=q_{2}, \quad q_{2 \tau_{-2}}=\frac{1}{2} p_{2}
$$

In our Hamiltonian formulation, this should be generated by the function $h_{-2}=\frac{1}{2} c_{2}$. Substituting these into (44) gives

$$
b_{11}=b_{22}=0 \quad, \quad b_{12}=-b_{21}=-\frac{1}{2} .
$$

The Hamiltonian operator $\mathbf{B}$ then takes the form

$$
\mathbf{B}=\mathbf{B}_{1}-\lambda \mathbf{B}_{0}
$$


where

$$
\begin{aligned}
\mathbf{B}_{0}=\left(\begin{array}{ccccc}
0 & 0 & 1 & 0 & 0 \\
0 & 0 & 0 & 1 & 0 \\
-1 & 0 & 0 & 0 & 0 \\
0 & -1 & 0 & 0 & 0 \\
0 & 0 & 0 & 0 & 2 \partial
\end{array}\right), \\
\mathbf{B}_{1}=\left(\begin{array}{cccccc}
0 & -\frac{1}{2} & 4 q_{1} & & 0 & 2 q_{2} \\
\frac{1}{2} & 0 & -4 q_{2} & & -6 q_{1} & p_{2} \\
-4 q_{1} & 4 q_{2} & -2 \partial & 60 q_{1}^{2}+2 p_{2} & \Omega_{1} \\
0 & 6 q_{1} & -60 q_{1}^{2}-2 p_{2} & 0 & 2 \partial+\Omega_{2} \\
-2 q_{2} & -p_{2} & -\Omega_{1} & 2 \partial-\Omega_{2} & 4\left(\partial q_{1}+q_{1} \partial\right)
\end{array}\right),
\end{aligned}
$$

and $\Omega_{1}=40 q_{1}^{3}-20 q_{2}^{2}-2 c_{2}, \Omega_{2}=-40 q_{1} q_{2}-2 p_{1}$.

The modified fifth-order hierarchy has a single local Hamiltonian structure (almost identical to $\mathbf{B}_{0}$ above). This simple operator is transformed onto $\mathbf{B}_{1}-\lambda \mathbf{B}_{0}$ by the Miura map (12), when extended to this 5-dimensional space. Once again, this is the simplest proof of the fact that $\mathbf{B}_{0}$ and $\mathbf{B}_{1}$ are both Hamiltonian and compatible. The modified fifth-order $\mathrm{KdV}$ equation is

$$
v_{t_{2}}=-\frac{1}{2} \tilde{c}_{2 x}, \quad \tilde{c}_{2}=-2 v_{x x x x}+20 v^{2} v_{x x}+20 v v_{x}^{2}-12 v^{5}-5 k^{2}\left(v_{x x}-2 v^{3}\right)-\frac{15}{4} k^{4} v
$$

Comparing with (15), we have

$$
\tilde{c}_{2}=\alpha_{(2) x}-2 v \alpha_{(2)} .
$$

Using the expansion (13), the coordinates (32), and their $\xi$-derivatives, we obtain an infinite expansion for $\tilde{c}_{2}$. This is our $h$, from which $h_{(r)}=\left(\lambda^{r+2} h\right)_{+}$is calculated.

The time evolution (26) is calculated from $h_{(r)}$ by (39) (compare with (31)):

$$
\gamma=\delta_{p_{1}} h_{(r)}, \quad \alpha=-2 \delta_{c_{2}} h_{(r)}, \quad \beta=\delta_{p_{2}} h_{(r)}+\left(2 q_{1}-\frac{1}{2} \lambda\right) \delta_{c_{2}} h_{(r)} .
$$

When $\xi$-derivatives of the phase space variables are zero, then $\mathbf{B}_{0}$ and $\mathbf{B}_{1}$ reduce to the previously known [5] Poisson matrices of the stationary fifth-order KdV equation. We then have just three independent Hamiltonians: $c_{2}, h_{-2}, h_{-1}$. The integral $c_{2}$ is the Casimir of $\mathbf{B}_{0}$, and $h_{-1}$ is the Casimir of $\mathbf{B}_{1}$ in this reduction.

\section{Some two-component examples}

We have illustrated our procedure in the context of the KdV hierarchy. The KdV equation itself is a special case, but the fifth-order member of the hierarchy already includes many of the general features. The details of the seventh-order KdV equation and its modification are presented in [16].

In this section, we present some interesting examples which illustrate certain additional features. For instance, Ito's equation requires non-zero $\mathbf{f}_{i}$ (see Remark (3.1)). 
4.1. The DWW hierarchy. This hierarchy is isospectral to a particular case of the energy dependent Schrödinger operator [3]. Since the KdV hierarchy is also a special case of this, the zero curvature representations are very similar:

$$
\begin{aligned}
& \left(\begin{array}{l}
\psi_{1} \\
\psi_{2}
\end{array}\right)_{x}=\left(\begin{array}{cc}
0 & 1 \\
\lambda^{2}-\lambda u_{1}-u_{0} & 0
\end{array}\right)\left(\begin{array}{l}
\psi_{1} \\
\psi_{2}
\end{array}\right) \equiv U \Psi, \\
& \left(\begin{array}{l}
\psi_{1} \\
\psi_{2}
\end{array}\right)_{t_{m}}=\left(\begin{array}{cc}
-\frac{1}{2} \alpha_{(m) x} & \alpha_{(m)} \\
C_{(m)} & \frac{1}{2} \alpha_{(m) x}
\end{array}\right)\left(\begin{array}{l}
\psi_{1} \\
\psi_{2}
\end{array}\right) \equiv V_{(m)} \Psi
\end{aligned}
$$

where $C_{(m)}=-\frac{1}{2} \alpha_{(m) x x}-\left(u_{0}+u_{1} \lambda-\lambda^{2}\right) \alpha_{(m)}$ and $\alpha_{(m)}=\sum_{i=0}^{m} \alpha_{m-i} \lambda^{i}$. The isospectral flows are tri-Hamiltonian, with

$$
\mathbf{u}_{t_{m}}=\mathbf{B}_{2} \delta_{u} \mathcal{H}_{m}=\mathbf{B}_{1} \delta_{u} \mathcal{H}_{m+1}=\mathbf{B}_{0} \delta_{u} \mathcal{H}_{m+2}
$$

where $\mathbf{u}=\left(u_{0}, u_{1}\right)^{T}$, and

$$
\mathbf{B}_{0}=\left(\begin{array}{cc}
-J_{1} & -J_{2} \\
-J_{2} & 0
\end{array}\right), \quad \mathbf{B}_{1}=\left(\begin{array}{cc}
J_{0} & 0 \\
0 & -J_{2}
\end{array}\right), \quad \mathbf{B}_{2}=\left(\begin{array}{cc}
0 & J_{0} \\
J_{0} & J_{1}
\end{array}\right),
$$

with $J_{0}=\frac{1}{2} \partial^{3}+2 u_{0} \partial+u_{0 x}, J_{1}=2 u_{1} \partial+u_{1 x}, J_{2}=-2 \partial$. The first few Hamiltonians are

$$
\begin{aligned}
& \mathcal{H}_{0}=u_{1}, \quad \mathcal{H}_{1}=u_{0}+\frac{1}{4} u_{1}^{2}, \quad \mathcal{H}_{2}=\frac{1}{2} u_{0} u_{1}+\frac{1}{8} u_{1}^{3}, \\
& \mathcal{H}_{3}=-\frac{1}{16} u_{1 x}^{2}+\frac{1}{4} u_{0}^{2}+\frac{3}{8} u_{0} u_{1}^{2}+\frac{5}{64} u_{1}^{4}, \\
& \mathcal{H}_{4}=-\frac{1}{8} u_{0 x} u_{1 x}-\frac{5}{32} u_{1} u_{1 x}^{2}+\frac{3}{8} u_{0}^{2} u_{1}+\frac{5}{16} u_{0} u_{1}^{3}+\frac{7}{128} u_{1}^{5} .
\end{aligned}
$$

The $t_{1}$ and $t_{2}$ flows are

$$
\begin{aligned}
& u_{0 t_{1}}=\frac{1}{4}\left(u_{1 x x x}+4 u_{0} u_{1 x}+2 u_{0 x} u_{1}\right), \\
& u_{1 t_{1}}=\left(u_{0}+\frac{3}{4} u_{1}^{2}\right)_{x} \\
& u_{0 t_{2}}=\frac{1}{4}\left(u_{0 x x x}+6 u_{0} u_{0 x}\right)+\frac{3}{8}\left(u_{1} u_{1 x x x}+3 u_{1 x} u_{1 x x}+u_{0 x} u_{1}^{2}+4 u_{0} u_{1} u_{1 x}\right), \\
& u_{1 t_{2}}=\frac{1}{4}\left(u_{1 x x}+6 u_{0} u_{1}+\frac{5}{2} u_{1}^{3}\right)_{x} .
\end{aligned}
$$

The first of these is known as the dispersive water wave (DWW) equation (after a simple change of coordinates) whilst the second is a coupled $\mathrm{KdV}$ equation. Half the flows reduce to those of the KdV hierarchy when $u_{1}=0$.

Example 4.1 (The DWW equation). The stationary solutions of the DWW equation (47) have Lagrangian

$$
\begin{aligned}
\mathcal{L} & =\mathcal{H}_{3}-u_{0} c_{1}-u_{1} c_{2}-\frac{1}{4} u_{1}^{2} c_{1} \\
& =-\frac{1}{16} u_{1 x}^{2}+\frac{1}{4} u_{0}^{2}+\frac{3}{8} u_{0} u_{1}^{2}+\frac{5}{64} u_{1}^{4}-u_{0} c_{1}-u_{1} c_{2}-\frac{1}{4} u_{1}^{2} c_{1}
\end{aligned}
$$


where the terms involving $c_{i}$ form the Casimirs of $\mathbf{B}_{0}$. As a function of $u_{0}$ and $u_{1}$, this is degenerate, but Lagrange's equations

$$
c_{1}=\frac{1}{2} u_{0}+\frac{3}{8} u_{1}^{2}, \quad c_{2}=\frac{1}{8} u_{1 x x}+\frac{3}{4} u_{0} u_{1}+\frac{5}{16} u_{1}^{3}-\frac{1}{2} u_{1} c_{1},
$$

can be used to eliminate $u_{0}$, resulting in a one degree of freedom system with Lagrangian

$$
\hat{\mathcal{L}}=-\frac{1}{8}\left(\frac{1}{2} u_{1 x}^{2}+\frac{1}{2} u_{1}^{4}-4 c_{1} u_{1}^{2}+8 c_{2} u_{1}\right) .
$$

Convenient coordinates for the stationary manifold of the DWW equation thus are

$$
q=u_{1}, \quad p=-\frac{1}{8} u_{1 x}, \quad c_{1}, \quad c_{2} \text {. }
$$

The DWW equation corresponds to $V_{(1)}$ (of (45)) with $\alpha_{(1)}=\lambda+\frac{1}{2} u_{1}$, and with $u_{0}$ and $u_{1 x x}$ eliminated using (49), so the $x-t$ reversed spectral problem is (33) with $\varphi: \mathbf{q} \mapsto \mathbf{b}$ given by

$$
a=2 p, \quad b=\lambda+\frac{1}{2} q, \quad c=\lambda^{3}-\frac{1}{2} \lambda^{2} q+\lambda\left(\frac{1}{4} q^{2}-2 c_{1}\right)-2 c_{2}+q c_{1}-\frac{1}{8} q^{3}
$$

with Jacobian

$$
\mathcal{J}_{\varphi}=\left(\begin{array}{cccc}
\frac{1}{2} & 0 & 0 & 0 \\
\gamma & 0 & q-2 \lambda & -2 \\
0 & 2 & 0 & 0
\end{array}\right)
$$

where $\gamma=-\frac{3}{8} q^{2}+c_{1}+\frac{1}{2} \lambda q-\frac{1}{2} \lambda^{2}$, whose right kernel is spanned by

$$
\mathbf{v}_{1}=\left(0,0,1, \frac{1}{2} q-\lambda\right)^{T} \text {. }
$$

Thus, with time evolution (45), we once again have the underdetermined system (34) for $\mathbf{q}_{\tau}$ and overdetermined system (35) for $\delta_{b} \tilde{h}$. One solution for $\delta_{b} \tilde{h}$ is given by

$$
\delta_{b} \tilde{h}=D \delta_{q} h \quad \text { with } \quad D \mathcal{J}_{\varphi}^{\dagger}=I_{3}
$$

where

$$
D=\left(\begin{array}{cccc}
2 & 0 & 0 & \gamma \\
0 & 0 & 0 & -\frac{1}{2} \\
0 & \frac{1}{2} & 0 & 0
\end{array}\right)
$$

Once again the $\mathbf{f}_{1}$ of (39) is zero, so $\hat{D}=D$. Our aim is to find a $4 \times 4$ matrix operator $\mathbf{B}$ satisfying (41). We use $D$ and $\overline{\mathbf{B}}$ of (27) to obtain a particular solution $\overline{\mathbf{B}}=D^{\dagger} \tilde{\mathbf{B}} D$

$$
\overline{\mathbf{B}}=\left(\begin{array}{cccc}
0 & -\frac{1}{2} q-\lambda & 0 & -4 p+\partial \\
\frac{1}{2} q+\lambda & -\frac{1}{8} \partial & 0 & -\Gamma \\
0 & 0 & 0 & 0 \\
4 p+\partial & \Gamma & 0 & \chi
\end{array}\right)
$$


where

$$
\begin{aligned}
& \Gamma=\frac{1}{8} q^{3}-\frac{1}{2} q c_{1}+\frac{1}{2} c_{2}, \\
& \chi=\partial\left(-\frac{3}{16} q^{2}+\frac{1}{2} c_{1}+\frac{1}{4} \lambda q\right)+\left(-\frac{3}{16} q^{2}+\frac{1}{2} c_{1}+\frac{1}{4} \lambda q\right) \partial-\frac{1}{2} \lambda^{2} \partial .
\end{aligned}
$$

To this we can add an extra term:

$$
\mathbf{B}=\overline{\mathbf{B}}+\mathbf{v}_{1} b_{11} \mathbf{v}_{1}^{\dagger}
$$

where $b_{11}$ is determined by comparison with the $\tau_{-1}$ flow: $b_{11}=\frac{1}{2} \partial$. We find

$$
\mathbf{B}=\mathbf{B}_{1}-\lambda \mathbf{B}_{0}
$$

with

$$
\begin{gathered}
\mathbf{B}_{0}=\left(\begin{array}{cccc}
0 & 1 & 0 & 0 \\
-1 & 0 & 0 & 0 \\
0 & 0 & 0 & \frac{1}{2} \partial \\
0 & 0 & \frac{1}{2} \partial & 0
\end{array}\right), \\
\mathbf{B}_{1}=\left(\begin{array}{cccc}
0 & -\frac{1}{2} q & 0 & -4 p+\partial \\
\frac{1}{2} q & -\frac{1}{8} \partial & 0 & -\Gamma \\
0 & 0 & \frac{1}{2} \partial & \frac{1}{4} \partial q \\
4 p+\partial & \Gamma & \frac{1}{4} q \partial & \partial \rho+\rho \partial
\end{array}\right),
\end{gathered}
$$

where $\rho=-\frac{1}{8} q^{2}+\frac{1}{2} c_{1}$.

The DWW equation is tri-Hamiltonian in any coordinate system. We form the recursion operator

$$
\mathbf{R}=\mathbf{B}_{1} \mathbf{B}_{0}^{-1}=\left(\begin{array}{cccc}
-\frac{1}{2} q & 0 & -8 p \partial^{-1}+2 & 0 \\
-\frac{1}{8} \partial & -\frac{1}{2} q & -2 \Gamma \partial^{-1} & 0 \\
0 & 0 & \frac{1}{2} \partial q \partial^{-1} & 1 \\
\Gamma & -4 p-\partial & 2 \rho+2 \partial \rho \partial^{-1} & \frac{1}{2} q
\end{array}\right)
$$

and then define $\mathbf{B}_{2}=\mathbf{R B}_{1}$ to give

$$
\mathbf{B}_{2}=\left(\begin{array}{cccc}
0 & \frac{1}{4} q^{2} & -4 p+\partial & \frac{1}{2}(\partial q-q \partial) \\
-\frac{1}{4} q^{2} & \frac{1}{16}(\partial q+q \partial) & -\Gamma & \frac{1}{2} \partial p-\frac{1}{8} \partial^{2} \\
4 p+\partial & \Gamma & \frac{1}{4}(\partial q+q \partial) & -\frac{1}{8} q^{2} \partial+\frac{1}{2}\left(\partial c_{1}+c_{1} \partial\right) \\
\frac{1}{2}(q \partial-\partial q) & \frac{1}{2} p \partial+\frac{1}{8} \partial^{2} & -\frac{1}{8} \partial q^{2}+\frac{1}{2}\left(\partial c_{1}+c_{1} \partial\right) & \frac{1}{2}\left(\partial c_{2}+c_{2} \partial\right)
\end{array}\right) .
$$

Remark 4.1. This construction is only possible on the PDE level since $\mathbf{B}_{0}$ becomes degenerate (and thus cannot be inverted) when reduced to the stationary case. Nevertheless, the reduced $\mathbf{B}_{2}$ is still valid. 
The hierarchy is tri-Hamiltonian with

$$
\mathbf{q}_{\tau_{r}}=\mathbf{B}_{2} \delta h_{r-1}=\mathbf{B}_{1} \delta h_{r}=\mathbf{B}_{0} \delta h_{r+1} .
$$

The integrals $h_{(r)}$ can be calculated as the fluxes of the DWW integrals in the usual $\left(u_{0}, u_{1}\right)$ coordinates, or can be calculated with a parameter dependent Miura map as previously explained. Parameter dependent Miura maps also enable us to prove that the operators $\mathbf{B}_{k}$ are Hamiltonian and compatible. The time evolution (45) is given in terms of $\delta h_{(r)}$ in a similar way to (31). The details can be found in [16]. Here we just give the parameter independent Miura maps and the modified Hamiltonian structures, together with the connecting diagram. The Miura maps,

$$
\begin{aligned}
& M_{1}\left\{\begin{array}{l}
q=2 \tilde{q}, \quad p=-\frac{1}{2} \tilde{q} \tilde{p}-\frac{1}{4} \tilde{c}_{1}, \quad c_{1}=\frac{1}{2} \tilde{c}_{2}, \\
c_{2}=-\frac{1}{2} \tilde{q}^{3}+\frac{1}{2} \tilde{q} \tilde{p}^{2}+\frac{1}{2} \tilde{q} \tilde{c}_{2}+\frac{1}{2} \tilde{p} \tilde{c}_{1}-\frac{1}{2} \tilde{p}_{\xi},
\end{array}\right. \\
& M_{2}\left\{\begin{array}{l}
\tilde{q}=2 \hat{p}, \quad \tilde{p}=\hat{q} \hat{p}-\frac{1}{2} \hat{c}_{1}, \quad \tilde{c}_{1}=-2 \hat{c}_{2}, \\
\tilde{c}_{2}=-\hat{q}^{2} \hat{p}^{2}+\hat{q} \hat{p} \hat{c}_{1}+\hat{q} \hat{c}_{2}+4 \hat{p}^{2}-\frac{1}{4} \hat{c}_{1}^{2}+\frac{1}{2} \hat{q}_{\xi},
\end{array}\right.
\end{aligned}
$$

transport Hamiltonian structures as shown in the figure below:

$$
\begin{aligned}
& q \longleftarrow \tilde{q} \longleftarrow \hat{q} \\
& \mathbf{B}_{2} \longleftarrow \tilde{\mathbf{B}}_{2} \longleftarrow \hat{\mathbf{B}}_{2} \\
& \mathbf{B}_{1} \longleftarrow \tilde{\mathbf{B}}_{1} \\
& \mathbf{B}_{0}
\end{aligned}
$$

where

$$
\begin{aligned}
& \tilde{\mathbf{B}}_{1}=\left(\begin{array}{cccc}
0 & 1 & 0 & 0 \\
-1 & 0 & 0 & 0 \\
0 & 0 & -2 \partial & 0 \\
0 & 0 & 0 & 2 \partial
\end{array}\right) \\
& \tilde{\mathbf{B}}_{2}=\left(\begin{array}{cccc}
0 & -\tilde{q} & 0 & 2 \tilde{q} \tilde{p}+\tilde{c}_{1}+\partial \\
\tilde{q} & 0 & \partial & 3 \tilde{q}^{2}-\tilde{p}^{2}-\tilde{c}_{2} \\
0 & \partial & 0 & -2 \partial \tilde{p} \\
-2 \tilde{q} \tilde{p}-\tilde{c}_{1}+\partial & -3 \tilde{q}^{2}+\tilde{p}^{2}+\tilde{c}_{2} & -2 \tilde{p} \partial & 2 \partial \tilde{q}+2 \tilde{q} \partial
\end{array}\right) \\
& \hat{\mathbf{B}}_{2}=\left(\begin{array}{cccc}
0 & 1 & 0 & 0 \\
-1 & 0 & 0 & 0 \\
0 & 0 & 0 & \partial \\
0 & 0 & \partial & 0
\end{array}\right)
\end{aligned}
$$

Remark 4.2. The other members of the DWW hierarchy are very similar, but the matrix operators are larger $(6 \times 6$ in the case of the third-order DWW equation (48)). 
4.2. The Ito hierarchy. We consider the isospectral flows of

$$
\left(\begin{array}{l}
\psi_{1} \\
\psi_{2}
\end{array}\right)_{x}=\left(\begin{array}{cc}
0 & 1 \\
\lambda-u_{1}-u_{0} \lambda^{-1} & 0
\end{array}\right)\left(\begin{array}{l}
\psi_{1} \\
\psi_{2}
\end{array}\right)
$$

The general time evolution is given by $V_{(m)}$ of (45), but with $C_{(m)}=-\frac{1}{2} \alpha_{(m) x x}-$ $\left(u_{0} \lambda^{-1}+u_{1}-\lambda\right) \alpha_{(m)}$. The $t_{1}$ flow, corresponding to $\alpha_{(1)}=\lambda+\frac{1}{2} u_{1}$, is the coupled $\mathrm{KdV}$ system

$$
u_{0 t_{1}}=\frac{1}{2} u_{0 x} u_{1}+u_{0} u_{1 x}, \quad u_{1 t_{1}}=\frac{1}{4}\left(u_{1 x x x}+6 u_{1} u_{1 x}+4 u_{0 x}\right) .
$$

In the coordinates $q=u_{1}, r=2 u_{0}^{1 / 2}$, this takes the form of Ito's equation. This system is tri-Hamiltonian [2]

$$
\mathbf{u}_{t_{m}}=\mathbf{B}_{2} \delta_{u} \mathcal{H}_{m}=\mathbf{B}_{1} \delta_{u} \mathcal{H}_{m+1}=\mathbf{B}_{0} \delta_{u} \mathcal{H}_{m+2}
$$

where $\mathbf{B}_{k}$ takes the same form as (46), but with

$$
J_{0}=2 u_{0} \partial+u_{0 x}, \quad J_{1}=\frac{1}{2} \partial^{3}+2 u_{1} \partial+u_{1 x}, \quad J_{2}=-2 \partial .
$$

The first few Hamiltonians are

$$
\begin{aligned}
& \mathcal{H}_{0}=u_{1}, \quad \mathcal{H}_{1}=u_{0}+\frac{1}{4} u_{1}^{2}, \quad \mathcal{H}_{2}=-\frac{1}{16} u_{1 x}^{2}+\frac{1}{2} u_{0} u_{1}+\frac{1}{8} u_{1}^{3}, \\
& \mathcal{H}_{3}=\frac{1}{8} u_{0} u_{1 x x}+\frac{1}{64} u_{1 x x}^{2}-\frac{5}{32} u_{1} u_{1 x}^{2}+\frac{1}{4} u_{0}^{2}+\frac{3}{8} u_{0} u_{1}^{2}+\frac{5}{64} u_{1}^{4} .
\end{aligned}
$$

Example 4.2 (Ito's equation). Ito's equation (50) corresponds to $\mathbf{B}_{0} \delta \mathcal{H}_{3}$. For the stationary flow, we need the kernel of $\mathbf{B}_{0}$, which is $\left(c_{1}, \frac{1}{2} u_{1} c_{1}+c_{2}\right)^{T}$ where $c_{i}$ are constants. Allowing $c_{i}$ to be non-constant, this is still a good representation for the gradient, even in the non-stationary case. Putting $\delta \mathcal{H}_{3}=\left(c_{1}, \frac{1}{2} u_{1} c_{1}+c_{2}\right)^{T}$,

$$
\begin{aligned}
& \frac{1}{8}\left(u_{1 x x}+3 u_{1}^{2}+4 u_{0}\right)=c_{1}, \\
& \frac{1}{32}\left(u_{1 x x x x}+4 u_{0 x x}\right)+\frac{5}{32}\left(u_{1 x}^{2}+2 u_{1} u_{1 x x}+2 u_{1}^{3}\right)+\frac{3}{4} u_{0} u_{1}=c_{2}+\frac{1}{2} u_{1} c_{1},
\end{aligned}
$$

the second of which can be simplified to give

$$
c_{2}=\frac{1}{4} c_{1 x x}-\frac{1}{32} u_{1 x}^{2}+\frac{1}{4} u_{0} u_{1}-\frac{1}{16} u_{1}^{3}+\frac{1}{2} u_{1} c_{1} .
$$

The stationary flow of Ito's equation (50) is reduced to one degree of freedom by eliminating $u_{0}$ and is Lagrangian with

$$
\mathcal{L}=\frac{1}{32} u_{1} u_{1 x}^{2}-\frac{1}{16} u_{1}^{4}+\frac{1}{2} u_{1}^{2} c_{1}-u_{1} c_{2}
$$

where the $c_{i}$ are constants. This, up to an exact derivative, corresponds, after eliminating $u_{0}$, to $\mathcal{H}_{3}-u_{0} c_{1}-u_{1} c_{2}-\frac{1}{4} u_{1}^{2} c_{1}$. The term $u_{0} c_{1}+u_{1} c_{2}+\frac{1}{4} u_{1}^{2} c_{1}$ is the general Casimir of $\mathbf{B}_{0}$. Convenient coordinates for the stationary manifold of Ito's equation are thus

$$
q=u_{1}, \quad p=\frac{1}{16} u_{1} u_{1 x}, \quad c_{1}, \quad c_{2}
$$


In the definition of $\mathcal{U}=V_{(1)}$, we thus can use (51), (52) to eliminate $u_{0}$ and $u_{1 x x}$ to give the $x-t$ reversed spectral problem as (33) with $\varphi: \mathbf{q} \mapsto \mathbf{b}$ given by

$$
\begin{aligned}
& a=-4 p q^{-1}, \quad b=\lambda+\frac{1}{2} q, \\
& c=\lambda^{2}-\frac{1}{2} \lambda q+\frac{1}{4} q^{2}-2 c_{1}+\left(q c_{1}-2 c_{2}-\frac{1}{8} q^{3}-16 p^{2} q^{-2}+4\left(p q^{-1}\right)_{\xi}\right) \lambda^{-1},
\end{aligned}
$$

where the term $4\left(p q^{-1}\right)_{\xi}$ stems from $c_{1 x x}=\frac{1}{2} u_{1 \xi x}=8\left(p q^{-1}\right)_{\xi}$

The Jacobian $\mathcal{J}_{\varphi}$ is the Fréchet derivative of this map:

$$
\mathcal{J}_{\varphi}=\left(\begin{array}{cccc}
\frac{1}{2} & 0 & 0 & 0 \\
\sigma & \lambda^{-1}\left(-32 p q^{-2}+4 \partial q^{-1}\right) & \lambda^{-1} q-2 & -2 \lambda^{-1} \\
4 p q^{-2} & -4 q^{-1} & 0 & 0
\end{array}\right)
$$

where

$$
\sigma=\lambda^{-1}\left(-\frac{3}{8} q^{2}+c_{1}+32 p^{2} q^{-3}-4 \partial p q^{-2}\right)+\frac{1}{2} q-\frac{1}{2} \lambda .
$$

The right kernel of $\mathcal{J}_{\varphi}$ is spanned by

$$
\mathbf{v}_{1}=\left(0,0,1, \frac{1}{2} q-\lambda\right)^{T}
$$

Thus, with this time evolution, we once again have the underdetermined system (34) for $\mathbf{q}_{\tau}$ and overdetermined system (35) for $\delta_{b} \tilde{h}$. One solution for $\delta_{b} \tilde{h}$ is given by

$$
\delta_{b} \tilde{h}=D \delta_{q} h \quad \text { with } \quad D \mathcal{J}_{\varphi}^{\dagger}=I_{3}
$$

where

$$
D=\left(\begin{array}{cccc}
2 & 2 p q^{-1} & 0 & -\frac{3}{8} q^{2}+c_{1}+\frac{1}{2} \lambda q-\frac{1}{2} \lambda^{2} \\
0 & 0 & 0 & -\frac{1}{2} \lambda \\
0 & -\frac{1}{4} q & 0 & 4 p q^{-1}+\frac{1}{2} \partial
\end{array}\right)
$$

To this $D$ we are free to add the transpose of an element of the right kernel of $\mathcal{J}_{\varphi}$, since this does not change the relationship (35):

$$
\delta_{b} \tilde{h}=\left(D+\mathbf{f}_{1} \mathbf{v}_{1}^{\dagger}\right) \delta_{q} h \equiv \hat{D} \delta_{q} h .
$$

However, in this case, $\mathbf{f}_{1} \neq 0$ (see Remark (3.1)), as we find by substituting the $\tau_{-1}$ flow (stationary Ito's equation) into

$$
\mathcal{J}_{\varphi} \mathbf{q}_{\tau_{r}}=\tilde{\mathbf{B}} \hat{D} \delta_{q} h_{(r)}
$$

We find

$$
\mathbf{f}_{1}=\left(\Upsilon_{1},-\frac{1}{2}, 0\right)^{T}
$$

where

$$
\Upsilon_{1}=\lambda^{-1} \theta+\frac{1}{2} q-\frac{1}{2} \lambda, \quad \text { with } \quad \theta=\frac{1}{8} q^{2}-c_{1}+2 c_{2} q^{-1}+16 p^{2} q^{-3}-4 q^{-1}\left(p q^{-1}\right)_{\xi}
$$


after which

$$
\hat{D}=\left(\begin{array}{cccc}
2 & 2 p q^{-1} & \Upsilon_{1} & \Upsilon_{2} \\
0 & 0 & -\frac{1}{2} & -\frac{1}{4} q \\
0 & -\frac{1}{4} q & 0 & 4 p q^{-1}+\frac{1}{2} \partial
\end{array}\right)
$$

where

$$
\Upsilon_{2}=\left(\frac{1}{2} \lambda^{-1} q-1\right) \theta-\frac{1}{8} q(q+2 \lambda)+c_{1} .
$$

We then can calculate $\overline{\mathbf{B}}=D^{\dagger} \tilde{\mathbf{B}} D$ :

$$
\overline{\mathbf{B}}=\left(\begin{array}{cccc}
0 & \frac{1}{4} q^{2}+\frac{1}{2} \lambda q & 8 p q^{-1}+\partial & \frac{1}{2} \partial q-\frac{1}{2} q \partial-8 \lambda p q^{-1}-\lambda \partial \\
-\frac{1}{4} q^{2}-\frac{1}{2} \lambda q & -\frac{1}{32} q \partial q & \Omega_{1}+\partial p q^{-1} & \frac{1}{16} q \partial^{2}+\frac{1}{2} \partial p-\lambda \Omega_{1}-\lambda \partial p q^{-1} \\
\partial-8 p q^{-1} & p q^{-1} \partial-\Omega_{1} & \Gamma_{5} & \Omega_{2}\left(\frac{1}{2} q-\lambda\right)+\rho_{1} \partial+\partial \rho_{2} \\
\Gamma_{1} & \Gamma_{2} & \Gamma_{3} & \Gamma_{4}
\end{array}\right)
$$

where

$$
\begin{gathered}
\Gamma_{1}=-\frac{1}{2} \partial q+\frac{1}{2} q \partial+8 \lambda p q^{-1}-\lambda \partial, \quad \Gamma_{2}=-\frac{1}{16} \partial^{2} q+\frac{1}{2} p \partial+\lambda \Omega_{1}-\lambda p q^{-1} \partial \\
\Gamma_{3}=\left(\frac{1}{2} q-\lambda\right) \Omega_{2}+\partial \rho_{1}+\rho_{2} \partial, \quad \Gamma_{4}=\left(\frac{1}{2} q-\lambda\right) \Omega_{2}\left(\frac{1}{2} q-\lambda\right)+\partial \rho_{3}+\rho_{3} \partial+\frac{1}{8} \partial^{3} \\
\Gamma_{5}=\Omega_{2}+\frac{1}{4}(\partial q+q \partial)-\frac{1}{2} \lambda \partial \\
\rho_{1}=-\frac{1}{8} q^{2}+\frac{1}{2} c_{1}, \quad \rho_{2}=\frac{1}{2} c_{1}-\frac{1}{4} \lambda q, \quad \rho_{3}=\frac{1}{2} c_{2}+\frac{1}{8} \lambda q^{2}-\frac{1}{2} \lambda c_{1}, \\
\Omega_{1}=q \rho_{1}-\frac{1}{2} c_{2}+4 p^{2} q^{-2}, \quad \Omega_{2}=\frac{1}{2} \lambda^{-1}(\partial \theta+\theta \partial) .
\end{gathered}
$$

To this, we add the extra term

$$
\mathbf{B}=\overline{\mathbf{B}}+\mathbf{v}_{1} b_{11} \mathbf{v}_{1}^{\dagger}
$$

where $b_{11}$ is determined by comparison with the $\tau_{-1}$ flow: $b_{11}=-\Omega_{2}$. We find $\mathbf{B}=\mathbf{B}_{2}-\lambda \mathbf{B}_{1}$ where

$$
\begin{gathered}
\mathbf{B}_{1}=\left(\begin{array}{cccc}
0 & -\frac{1}{2} q & 0 & 8 p q^{-1}+\partial \\
\frac{1}{2} q & 0 & 0 & \Omega_{1}+\partial p q^{-1} \\
0 & 0 & \frac{1}{2} \partial & \frac{1}{4} \partial q \\
-8 p q^{-1}+\partial & -\Omega_{1}+p q^{-1} \partial & \frac{1}{4} q \partial & \partial \rho_{1}+\rho_{1} \partial
\end{array}\right) \\
\mathbf{B}_{2}=\left(\begin{array}{cccc}
0 & \frac{1}{4} q^{2} & 8 p q^{-1}+\partial & \frac{1}{2} \partial q-\frac{1}{2} q \partial \\
-\frac{1}{4} q^{2} & -\frac{1}{32} q \partial q & \Omega_{1}+\partial p q^{-1} & \frac{1}{16} q \partial^{2}+\frac{1}{2} \partial p \\
-8 p q^{-1}+\partial & -\Omega_{1}+p q^{-1} \partial & \frac{1}{4}(\partial q+q \partial) & \rho_{1} \partial+\frac{1}{2} \partial c_{1} \\
-\frac{1}{2} \partial q+\frac{1}{2} q \partial & -\frac{1}{16} \partial^{2} q+\frac{1}{2} p \partial & \partial \rho_{1}+\frac{1}{2} c_{1} \partial & \frac{1}{2}\left(\partial c_{2}+c_{2} \partial\right)+\frac{1}{8} \partial^{3}
\end{array}\right) .
\end{gathered}
$$


The notation anticipates the same phenomenon as in the $\left(u_{0}, u_{1}\right)$ coordinates. That is, the third Hamiltonian structure is actually the first! We define the recursion operators $\mathbf{R}=\mathbf{B}_{2} \mathbf{B}_{1}^{-1}$

$$
\mathbf{R}=\left(\begin{array}{cccc}
-\frac{1}{2} q & 0 & 16 p q^{-1} \partial^{-1}+2 & 0 \\
\frac{1}{16} q \partial & -\frac{1}{2} q & \left(2 \Omega_{1}+2 \partial p q^{-1}\right) \partial^{-1} & 0 \\
0 & 0 & \frac{1}{2} \partial q \partial^{-1} & 1 \\
-\Omega_{1}+\frac{1}{8} \partial^{2} & 8 p q^{-1}-\partial & 2 \rho_{1}+2 \partial \rho_{1} \partial^{-1} & \frac{1}{2} q
\end{array}\right)
$$

which is invertible. The Hamiltonian operator $\mathbf{B}_{0}$ then is constructed by $\mathbf{B}_{0}=\mathbf{R}^{-1} \mathbf{B}_{1}$

$$
\mathbf{B}_{0}=\left(\begin{array}{cccc}
0 & 1 & 0 & 0 \\
-1 & \frac{1}{8} \partial & 0 & 0 \\
0 & 0 & 0 & \frac{1}{2} \partial \\
0 & 0 & \frac{1}{2} \partial & 0
\end{array}\right)
$$

Remark 4.3. Once again, since $\mathbf{B}_{1}$ degenerates upon reduction to the stationary flow, this construction is only valid on the PDE level. However, $\mathbf{B}_{0}$ is still valid upon this reduction.

In the usual way, we can construct an infinite sequence of Hamiltonians satisfying the bi-Hamiltonian ladder, Miura maps and modified systems, and all the other formulae, such as (31), in a systematic way [16].

\section{Conclusions}

In this paper, we have been interested in the relationship between an integrable nonlinear evolution equation (PDE) and its stationary flow. We were particularly interested in the reduction of the infinite dimensional Hamiltonian structures to their finite dimensional counterparts. This reduction is most transparent when the PDE is written as a flow in a larger space whose coordinates are those of the stationary manifold, together with their $t$-derivatives.

Starting from a zero-curvature representation (reversing the roles of $U$ and $V$ ), we gave a systematic construction of the isospectral flows and their Hamiltonian structures. We have adopted the approach presented in $[3,4]$, which simultaneously constructs the isospectral flows, time evolutions of the wave functions, the Hamiltonians, and Hamiltonian structures. This close relationship between the zero curvature representation and the Hamiltonian formulation of the equations perhaps is best seen by the formula (31).

An unsatisfactory feature of our approach is that each stationary manifold has to be treated separately. Thus we construct $1 \times 1,3 \times 3,5 \times 5$, etc. Hamiltonian representations of the KdV equation, but know of no way of reducing from higher to lower order representations. This deficiency perhaps could be overcome by giving some Lie algebraic description of stationary reductions (see [21] for the general approach). However, since the appropriate spectral problems are such deep reductions of the generic case, we can expect the appropriate algebra to be rather complicated.

Whilst the importance of our results is mainly in the realm of the stationary reductions, we have, in passing, answered a number of the questions raised in [13], where 
they believed they had found a new hierarchy of equations. We have seen that, in fact, this hierarchy is just the KdV hierarchy in disguise.

In this paper, we presented a selection of examples, including the $\mathrm{KdV}, \mathrm{DWW}$, and Ito hierarchies. Further examples from the DWW and Boussinesq hierarchies can be found in [16] and will be presented elsewhere.

\section{Appendix: Generalised Lagrangians}

We consider a (generalised) Lagrangian:

$$
\mathcal{L}\left(q^{(0)}, q^{(1)}, \ldots, q^{(n)}\right), \quad q^{(i)}=\frac{d^{i} q}{d x^{i}}, \quad n \geq 1,
$$

where $\mathcal{L}$ is non-degenerate $\left(\frac{\partial^{2} \mathcal{L}}{\partial q^{(n) 2}} \neq 0\right)$. The corresponding Euler-Lagrange equations are

$$
\sum_{i=0}^{n}(-\partial)^{i} \frac{\partial \mathcal{L}}{\partial q^{(i)}}=0
$$

Canonical coordinates can be defined as

$$
\begin{aligned}
q_{i} & =q^{(i-1)}, \quad i=1, \ldots, n, \\
p_{n} & =\frac{\partial \mathcal{L}}{\partial q^{(n)}}, \quad p_{i}=\frac{\partial \mathcal{L}}{\partial q^{(i)}}-\dot{p}_{i+1}, \quad i=1, \ldots, n-1 .
\end{aligned}
$$

The Euler-Lagrange equations then take canonical Hamiltonian form with

$$
h=\sum_{i=1}^{n-1} q_{i+1} p_{i}+q_{n x} p_{n}-\mathcal{L}\left(q_{1}, \ldots, q_{n}, q_{n x}\right),
$$

where $q_{n x}$ is removed by inverting the non-degenerate Legendre transformation [22]. The generalisation to more than one component $q_{i}$ is straightforward.

Acknowledgements. Simon Harris was supported by a studentship of the SERC.

\section{References}

1. M. Antonowicz and M. Blaszak, On a non-standard Hamiltonian description of NLEE. In: Nonlinear Evolution Equations and Dynamical Systems (Eds. S. Carillo and O. Ragnisco), SpringerVerlag, Berlin, 1990, pp.152-156.

2. M. Antonowicz and A. P. Fordy, Coupled KdV equations with multi-Hamiltonian structures, Physica 28D (1987), 345-357.

3. —_ Factorisation of energy dependent Schrödinger operators: Miura maps and modified systems, Commun. Math. Phys. 124 (1989), 465-486.

4. __ Hamiltonian structures of nonlinear evolution equations. In: Soliton Theory : A Survey of Results (Ed. A. P. Fordy), Manchester University Press, Manchester, 1990, pp.273-312.

5. M. Antonowicz, A. P. Fordy, and S. Wojciechowski, Integrable stationary flows: Miura maps and bi-Hamiltonian structures, Phys. Letts. A 124 (1987), 143-150.

6. S. Baker, V. Z. Enolskii, and A. P. Fordy, Integrable quartic potentials and coupled KdV equations, Phys. Letts. A 201 (1995), 167-174.

7. O. I. Bogoyavlenskii and S. P. Novikov, The relationship between Hamiltonian formalisms of stationary and nonstationary problems, Func. Anal. Appls. 10 (1976), 8-11.

8. H. Flaschka, Relations between infinite-dimensional and finite-dimensional isospectral equations. In: Nonlinear Integrable Systems. Classical Theory and Quantum Theory (Eds. M. Jimbo and T. Miwa), World Scientific, Singapore, 1983. 
9. A. P. Fordy, Isospectral flows: their Hamiltonian structures, Miura maps and master symmetries. In: Solitons in Physics, Mathematics, and Nonlinear Optics (Eds. P. J. Olver and D. L. Sattinger), Springer, NY, 1990, pp.97-121.

10. A. P. Fordy, editor, Soliton Theory: A Survey of Results, Manchester University Press, Manchester, 1990.

11. A. P. Fordy and J. Gibbons, Factorization of operators I: Miura transformations, J. Math. Phys. 21 (1980), 2508-2510.

12. _ Factorization of operators II, J. Math. Phys. 22 (1981), 1170-1175.

13. B. Fuchssteiner and W. Oevel, New hierarchies of nonlinear completely integrable systems related to a change of variables for evolution parameters, Physica 145A (1987), 67-95.

14. C. S. Gardner, The Korteweg-de Vries equation and generalizations. IV. The Korteweg-de Vries equation as a Hamiltonian system, J. Math. Phys. 12 (1971), 1548-1551.

15. I. M. Gelfand and I. Ya. Dorfman, Hamiltonian operators and algebraic structures related to them, Func. Anal. Appls. 13 (1979), 13-30.

16. S. D. Harris, Integrable nonlinear evolution equations and their stationary flows, $\mathrm{PhD}$ thesis, University of Leeds, 1994.

17. B. A. Kupershmidt and G. Wilson, Modifying Lax equations and the second Hamiltonian structure, Invent. Math. 62 (1981), 403-36.

18. F. Magri, A simple model of the integrable Hamiltonian equation, J. Math. Phys. 19 (1978), 1156-1162.

19. R. M. Miura, Korteweg-de Vries equation and generalizations I. A remarkable explicit nonlinear transformation, J. Math. Phys. 9 (1968), 1202-1204.

20. O. I. Mokhov, On the Hamiltonian property of an arbitrary evolution system on the set of stationary points of its integral, Math. USSR Izvestiya 31 (1988), 657-664.

21. A. G. Reyman and M. A. Semenov-Tian-Shansky, Group-theoretical methods in the theory of finite dimensional integrable systems. In: Dynamical Systems VII (Eds. V. I. Arnol'd and S. P. Novikov), pp.116-225. Springer-Verlag, Berlin, 1991.

22. E. T. Whittaker, A Treatise on the Analytical Dynamics of Particles and Rigid Bodies, Cambridge University Press, Cambridge, UK, 1988.

23. V. E. Zakharov and L. D. Faddeev, The Korteweg-de Vries equation as a completely integrable Hamiltonian system, Func. Anal. Appls. 5 (1971), 18-27.

Department of Applied Mathematical Studies and Centre for Nonlinear Studies, UniverSITY OF LEEDS, LEEDS LS2 9JT, UK

E-mail: allan@amsta.leeds.ac.uk 